\title{
MATRIX POWERS OVER FINITE FIELDS
}

MARIA T. ACOSTA-DE-OROZCO

\author{
Department of Mathematics \\ Penn State University \\ Beaver Campus \\ Monaca, Pennsylvania 15061
}

and

\section{JAVIER GOMEZ-CALDERON}

Department of Mathematics

Penn State University

New Kensington Campus

New Kensington, Pennsylvania 15068

(Received May 23, 1991 and in revised form May 18, 1992)

\begin{abstract}
Let $G F(q)$ denote the finite field of order $q=p^{e}$ with $p$ odd. Let $M$ denote the ring of $2 \times 2$ matrices with entries in $G F(q)$. Let $n$ denote a divisor of $q-1$ and assume $2 \leq n$ and 4 does not divide $n$. In this paper, we consider the problem of determining the number of $n$-th roots in $M$ of a matrix $B \in M$. Also, as a related problem, we consider the problem of lifting the solutions of $X^{2}=B$ over Galois rings.
\end{abstract}

KEY WORDS AND PHRASES. Finite fields and matrix powers.

1991 AMS SUBJECT CLASSIFICATION CODES. Primary 15A33.

\section{INTRODUCTION.}

Let $G F(q)$ denote the finite field of order $q=p^{n}$ with $p$ odd. Let $M$ denote the ring of $2 \times 2$ matrices with entries in $G F(q)$. Let $n$ denote a positive divisor of $q-1$. In this paper, we consider the problem of determining the number $N=N(n, B)$ of $n-t$ th roots in $M$ of a matrix $B \in M$; i.e., the number of solutions in $M$ of the equation

$$
X^{n}=B
$$

Our present work generalizes a recent paper of Donovan [1] in which the quadratic equation $X^{2}=B$ is solved over the ring $M$.

As a related problem, we also consider the problem of lifting solutions of equation (1.1), for $n=2$, over Galois rings. The Galois ring of order $p^{r m}$, denoted by $G R\left(p^{r}, m\right)$, can be obtained as a Galois extension of $z_{p^{r}}$ of degree $m$. The reader can find further details about Galois rings in the reference [4].

If $B$ denotes a scalar matrix, a multiple of the identity matrix, then equation (1.1) is called "scalar equation ". Scalar equations have been already studied by Hodges in [2]. In particular, if 
$n=2$ and $B$ denotes the identity matrix, then the solutions of (1.1) are called "involutory matrices". Involutory matrices over either a finite field or a quotient ring of the rational integers have been extensively researched, with a detailed extension to all finite commutative rings given by McDonald in [5].

\section{OVER FINITE FIELDS.}

Let $G F(q)$ denote the finite field of order $q=p^{e}$ with $p$ odd. Let $M$ denote the ring of $2 \times 2$ matrices with entries in $G F(q)$ and let $G L$ denote its group of units. For each $B$ in $M$ let $S(B)$ and $[B]$ denote, respectively, the stabilizer and the conjugate class of $B$ defined by

$$
S(B)=\{A \in G L: A B=B A\}
$$

and

$$
[B]=\left\{A B A^{-1}: A \in G L\right\}
$$

Thus

$$
|[B]|=[G L: S(B)] .
$$

Now for the purpose of the present work we will need the following stabilizers:

$$
\begin{aligned}
& S\left(\left(\begin{array}{ll}
a & 0 \\
0 & a
\end{array}\right)\right)=G L(q) \\
& S\left(\left(\begin{array}{ll}
0 & 0 \\
1 & 0
\end{array}\right)\right)=\left\{\left(\begin{array}{ll}
x & 0 \\
y & x
\end{array}\right): x, y \in G F(q), x \neq 0\right\} \\
& S\left(\left(\begin{array}{ll}
a & 0 \\
0 & b
\end{array}\right)\right)=\left\{\left(\begin{array}{ll}
x & 0 \\
0 & y
\end{array}\right): x, y \in G F(q), x y \neq 0\right\}, \quad(a-b) a b \neq 0 \\
& S\left(\left(\begin{array}{ll}
0 & a \\
1 & 0
\end{array}\right)\right)=\left\{\left(\begin{array}{ll}
x & a y \\
y & x
\end{array}\right): x, y \quad G F(q), x^{2}-a y^{2} \neq 0\right\}, \quad a \neq 0
\end{aligned}
$$

We now give a series of lemmas from which our main result, Theorem 6, will follow.

LEMMA 1. Assume $T^{n}=B$ for some $T$ and some non-scalar $B$ in $M$. Then $S(T)=S(B)$.

PROOF. Since $B$ is non-scalar, the minimal polynomial of $T$ is a quadratic polynomial $f_{T^{(x)}}=x^{2}+a x+b$. Therefore, $B=T^{n}=d T+e I$ for some constants $e$ and $0 \neq d$ in $G F(q)$. Thus, $S(T)=S(B)$.

LEMMA 2. If $n \geq 2$ then the number of matrices $T$ in $M$ so that $T^{n}=0$ is $q^{2}$.

PROOF. $T^{n}=0$ if and only if the minimal polynomial of $T$ is either $x$ or $x^{2}$. Hence, $T^{n}=0$ if and only if $T$ is similar to either $A=\left(\begin{array}{ll}0 & 0 \\ 0 & 0\end{array}\right)$ or $B=\left(\begin{array}{ll}0 & 0 \\ 1 & 0\end{array}\right)$. Therefore,

$$
\begin{aligned}
\left|\left\{T \in M: T^{n}=0\right\}\right| & =|[A]|+|[B]| \\
& =[G L: S(A)]+[G L: S(B)] \\
& =1+q(q-1)\left(q^{2}-1\right) /\left(q^{2}-q\right) \\
& =q^{2} .
\end{aligned}
$$

LEMMA 3. Let $2 \leq n$ denote a divisor of $q-1$ and assume that 4 does not divide $n$. For each $r$ in $G F(q)^{*}$ the number of distinct matrices $T$ in $M$ such that $T^{n}=\operatorname{diag}(r, r)$ is given by 
a) $n+\left(q^{2}-q\right)(n-1) n / 2 \quad$ if $\quad r \in G F(q)^{n}=\left\{y^{n}: y \in G F(q)\right\}$

b) $\left(q^{2}-q\right) n / 2 \quad$ if $r \notin G F(q)^{n} \quad$ but $r^{2} \in G F(q)^{n}$

c) 0

if $r^{2} \notin G F(q)^{n}$

PROOF. Let $w$ denote a primitive element of $G F(q)$ and write $r=w^{m}$ for some integer $1 \leq m \leq q-1$. Then $T^{n}=\operatorname{diag}(r, r)$ if and only if the minimal polynomial of $T$ divides $f(x)=x^{n}-w^{m}$. Now, if $D=(n, m)$ denotes the greatest common divisor of $n$ and $m$, then we obtain

$$
\begin{aligned}
f(x) & =\left(x^{n / D}\right)^{D}-\left(w^{m / D}\right) D \\
& =\prod_{i=0}^{D-1}\left(x^{n / D}-w^{(q-1) i / D+m / D}\right) \\
& =\prod_{i=0}^{D-1} h_{i}(x)
\end{aligned}
$$

We also see that $w^{(q-1) i / D+m / D}$ does not belong to $G F(q)^{s}$ for every odd prime factor $s$ of $n / D$. Therefore, by [3, ch. VIII, Th. 16], $h_{i}(x)$ is irreducible over $G F(q)$ for all $i$. Thus, $n / D=1$, $n / D=2$ or there are no matrices $T$ so that $T^{n}=\operatorname{diag}(r, r)$.

CASE 1: $n / D=1$. Then $n$ divides $m$ and $T^{n}=\operatorname{diag}(r, r)$ if and only if the minimal polynomial of $T$ is either $x-a$ or $(x-a)(x-b)$ where $a$ and $b$ denote two distinct roots in $G F(q)$ of the equation $x^{n}=r$. Hence, $T^{n}=\operatorname{diag}(r, r)$ if and only if $T$ is similar to either $A=\operatorname{diag}(a, a)$ or $B=\operatorname{diag}(a, b)$. Therefore,

$$
\begin{aligned}
\mid\left\{T \in M: T^{n}\right. & \left.=\operatorname{diag}(r, r)\}|=n|[A] \mid+\int_{2}^{n}\right)|[B]| \\
& =n+\left(\int_{2}^{n}\right) \frac{q\left(q-1\left(q^{2}-1\right)\right.}{(q-1)^{2}} \\
& =n+\left(q^{2}+q\right)(n-1) n / 2
\end{aligned}
$$

CASE 2: $n / D=2$. Then $n / 2$ divides $m$ and $T^{n}=\operatorname{diag}(r, r)$ if and only if the minimal polynomial of $T$ is a quadratic irreducible polynomial of the form $x^{2}-c$ where $c$ denotes a root of the equation $x^{n / 2}=r$. Thus, $T^{n}=\operatorname{diag}(r, r)$ if and only if $T$ is similar to $A=\left(\begin{array}{ll}0 & c \\ 1 & 0\end{array}\right)$. Therefore,

$$
\left|\left\{T \in M: T^{n}=\operatorname{diag}(r, r)\right\}\right|=\frac{q(q-1)\left(q^{2}-1\right) n}{\left(q^{2}-1\right)(2)}
$$

if $r \notin G F(q)^{n}$ but $r^{2} \in G F(q)^{n}$.

LEMMA 4. If $T^{n}=\operatorname{diag}(h, k)$ with $h \neq k$, then $T=\operatorname{diag}(r, s)$ for some $r$ and $s$ in $G F(q)$.

PROOF. Let $f(x)=x^{2}+a x+b$ denote the minimal polynomial of $T$. So, $T^{2}=-a T-b I$ and $c T+e I=\operatorname{diag}(h, k)$ for some $c$ and $e$ in $G F(q)$. Therefore, $T=\operatorname{diag}(r, s)$ for some $r$ and $s$ in $G F(q)$.

LEMMA 5. A non-scalar $2 \times 2$ diagonalizable matrix over $G F(q)$ is a $n-t h$ power in $M$ if and only if its eigenvalues, necessarily distinct, are $n-t h$ powers in $G F(q)$.

PROOF. Assume $T$ to be non-scalar and diagonalizable so that for some matrix $P$ in $G L$, $P T P^{-1}=\operatorname{diag}(h, k)$ where $h \neq k$ are the eigenvalues of $T$. If $h$ and $k$ are $n-t h$ powers, say $h=r^{n}$ and $k=s^{n}$, then 


$$
T=P^{-1} \operatorname{diag}(h, k) P=P^{-1}(\operatorname{diag}(r, s))^{n} P=\left(P^{-1} \operatorname{diag}(r, s) P\right)^{n} .
$$

Conversely, suppose $T=N^{n}$ and $T$ is diagonalizable. Say $P^{-1} T P=\operatorname{diag}(h, k)$ where $h \neq k$ are the eigenvalues of $T$. Hence

$$
\operatorname{diag}(h, k)=P^{-1} T P=P^{-1} N^{n} P=\left(P^{-1} N P\right)^{n} .
$$

Therefore, by Lemma $4, P^{-1} N P=\operatorname{diag}(r, s)$ with $r^{n}=h$ and $k^{n}=s$.

THEOREM 6. Let $B$ denote an element of $M$. Let $n$ denote a divisor of $q-1$. Assume $2 \leq n$ and 4 does not divide $n$. Then $B$ has

(a) more than $n^{2} n-t h$ roots in $M$ if and only if $B=r I$ for some $r$ in $G F(q)$ so that $r^{2} \in G F(q)^{n}$.

(b) exactly $n^{2}$ distinct $n-t h$ roots in $M$ if and only if $B$ has unequal nonzero eigenvalues which are $n$-th powers in $G L(q)$.

(c) at most $n$ distinct roots in $M$, otherwise.

PROOF. If $B=r I$ for some $r$ in $G F(q)$, then, by Lemma 3, $T$ has

(i) more than $n^{2} n-t h$ roots if and only if $r^{2} \in G F(q)^{n}$ and

(ii) zero $n-t h$ roots if and only if $r^{2} \notin G F(q)^{n}$.

We now assume that $T$ is non-scalar.

CASE 1: $B$ diagonalizable. Then by Lemma $5, B$ is a $n-t h$ power in $M$ if and only if its eigenvalues, necessarily distinct, are $n-t h$ powers in $G F(q)$. Therefore, $B$ has exactly (iii) $n^{2}$ distinct $n-t h$ roots in $M$ if and only if $B$ has unequal nonzero eigenvalues which are $n-t h$ powers in $G F(q)$ and

(iv) zero $n-t h$ roots otherwise.

CASE 2: $B$ non-diagonalizable. Then the minimal polynomials of both $B$ and $T$ are either: quadratic irreducible or quadratic perfect square polynomials. We also see that if $T^{n}=B$ then the minimal polynomial of $T$ is a factor of $f_{B}\left(x^{n}\right)$ where $f_{B}(x)$ denotes the minimal polynomial of $B$. Therefore, there are at most $n$ possible minimal polynomial $f_{T}(x)$. Further, $\left(P^{-1}{ }_{T P}\right)^{n}=B$ if and only if $P \in S(B)$. Therefore, since $[S(B): S(T)]=1$ by Lemma $1, B$ has at most $n$ distinct $n-t h$ roots in $M$.

\section{LIFTING SOLUTIONS.}

Let $G R\left(p^{r}, m\right)$ denote the Galois ring of order $p^{r m}$ with $p$ odd. For purposes of construction and ease of implementation of Galois rings, one can construct $G R\left(p^{r}, m\right)$ by considering $\left(z p^{r}\right)[x] /(f)$ where $f$ is a monic irreducible polynomial of degree $m \geq 1$ over the finite field $G F\left(p^{m}\right)=G F(q)$ with $p$ prime. Further details concerning properties of Galois rings can be found in the reference [4].

In this section, we will consider a special case, $n=2$, of lifting solutions over Galois rings. More specifically, we will prove the following

THEOREM 7. Let $M\left(p^{r+1}, m\right)$ denote the ring of all $2 \times 2$ matrices with entries in $G R\left(p^{r+1}, m\right)$. Let $A$ denote an element of $M$. Assume that $\bar{A}$, the reduction of $A$ modulo $p$, is a non-scalar invertible matrix in $M(p, m)$. Let $X_{\circ}=\left(\begin{array}{ll}a & b \\ c & d\end{array}\right) \in M\left(p^{r}, m\right)$ denote a solution of $X^{2}=A(\bmod$ $\left.p^{r}\right)$. Then $X_{\text {。 }}$ can be lifted from $M\left(p^{r}, m\right)$ to $M\left(p^{r+1}, m\right)$ in

(a) a unique way if $\overline{b c d} \neq 0$.

(b) $q=p^{m}$ different ways if either $\bar{d}=0$ or $\overline{c d} \neq 0$ and $\bar{b}=0$.

(c) $q^{2}=p^{2 m}$ different ways if $\bar{d} \neq 0$ and $\bar{c}=0$.

PROOF. Let $X=\left(\begin{array}{cc}x & y \\ z & w\end{array}\right)$ where $x, y, z$ and $w$ are elements of the field $G R(p, m)$ to be specified presently, then 


$$
\left(X_{\circ}+X p^{r}\right)^{2} \equiv X_{\circ}^{2}+\left(X_{0} X+X X_{\circ}\right) p^{r} \bmod p^{r+1}
$$

Now, since $X_{0}^{2}=A$ over $G R\left(p^{r}, m\right)$, we can write $X_{0}^{2}=A-C p^{r}$ for some $2 \times 2$ matrix $C$ over the ring $G R(p, m)$. Hence,

$$
\left(X_{\circ}+X p^{r}\right)^{2} \equiv A+\left(X_{\circ} X+X X_{\circ}-C\right) p^{r} \bmod p^{r+1}
$$

Therefore, $\left(X_{\circ}+X p^{r}\right)^{2}=A$ over the ring $G R\left(p^{r+1}, m\right)$, if and only if

$$
X_{\circ} X+X X_{\circ}=C
$$

over the field $G R(p, m)$; i.e., if and only if

$$
\left(\begin{array}{ll}
a & b \\
c & d
\end{array}\right)\left(\begin{array}{ll}
x & y \\
z & w
\end{array}\right)+\left(\begin{array}{ll}
x & y \\
z & w
\end{array}\right)\left(\begin{array}{ll}
a & b \\
c & d
\end{array}\right) \equiv\left(\begin{array}{ll}
c_{1} & c_{2} \\
c_{3} & c_{4}
\end{array}\right)(\bmod p)
$$

where $C=\left(\begin{array}{ll}c_{1} & c_{2} \\ c_{3} & c_{4}\end{array}\right)$

Hence, we have to count the number of solutions, in $G R(p, m)$, of the linear system

$$
\left(\begin{array}{cccc}
y & z & w & x \\
c & b & 0 & 2 a \\
a+d & 0 & b & b \\
0 & a+d & c & c \\
c & b & 2 d & 0
\end{array}\right) \equiv\left(\begin{array}{c}
c_{1} \\
c_{2} \\
c_{3} \\
c_{4}
\end{array}\right) \quad(\bmod p)
$$

or

$$
\left(\begin{array}{cccc}
y & z & w & x \\
c(a+d) & 0 & b c & b c \\
0 & a+d & c & c \\
0 & 0 & 2 b c d & -2 a b c \\
0 & 0 & 0 & E_{1}
\end{array}\right) \equiv\left(\begin{array}{c}
c_{1} c \\
c_{3} \\
\left(c_{4}-c_{1}\right) b c \\
E_{2}
\end{array}\right)
$$

where $E_{1}=2(a+d)(a d-b c)$ and $E_{2}=c_{1} a d+c_{1} d^{2}-c_{2} c d-b c_{3} d+c_{4} b c-c_{1} b c$. So, since $\bar{A}$ is non-scalar and invertible, $E_{1} \neq 0$. Therefore, a straightforward inspection of the above last augmented matrix will complete the proof of the theorem.

\section{REFERENCES}

1. DONOVAN, T.P., Matrix squares over $G F(q), q$ odd, Congressus Numerantium 66 (1988), $113-$ 122.

2. HODGES, J.H., Scalar polynomial equations for matrices over a finite field, Duke Math. J. 25 (1958), 291-296.

3. LANG, S., "Algebra", Addison-Wesley, Reading, Mass., 1971.

4. MCDONALD, B.R., "Finite Rings with Identity", Marcel Bekker, New York, 1974.

5. MCDONALD, B.R., Involutory matrices over finite local rings, Canadian Journal of Math. 24 (1972), 369-378. 


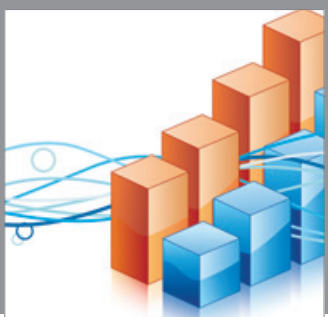

Advances in

Operations Research

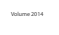

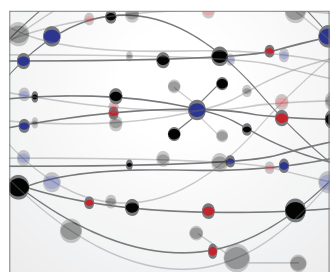

\section{The Scientific} World Journal
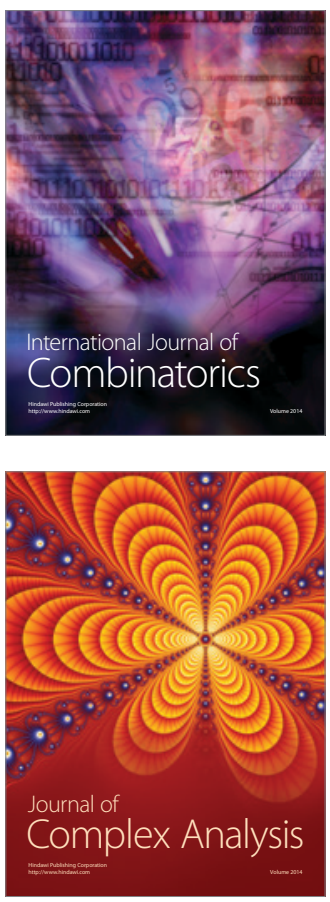

International Journal of

Mathematics and

Mathematical

Sciences
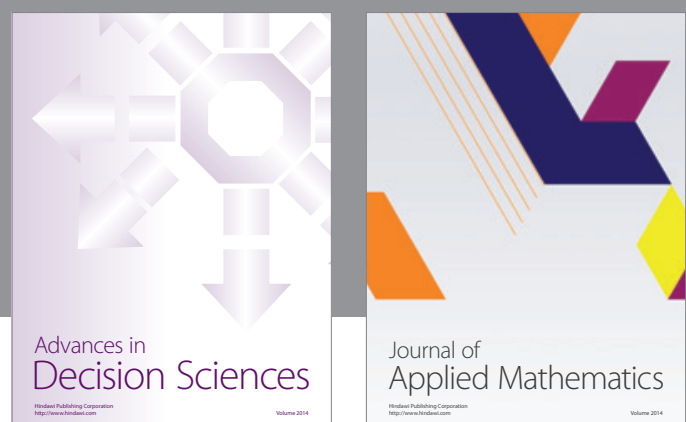

Journal of

Applied Mathematics
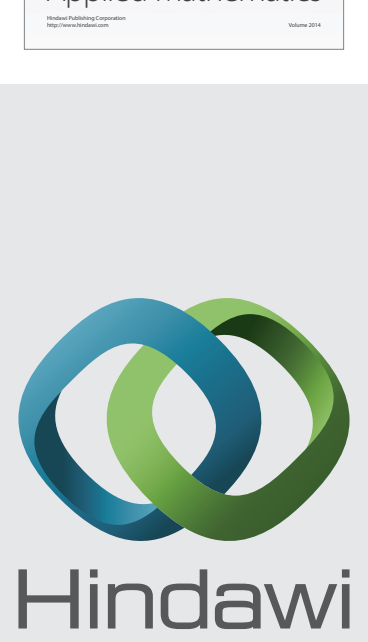

Submit your manuscripts at http://www.hindawi.com
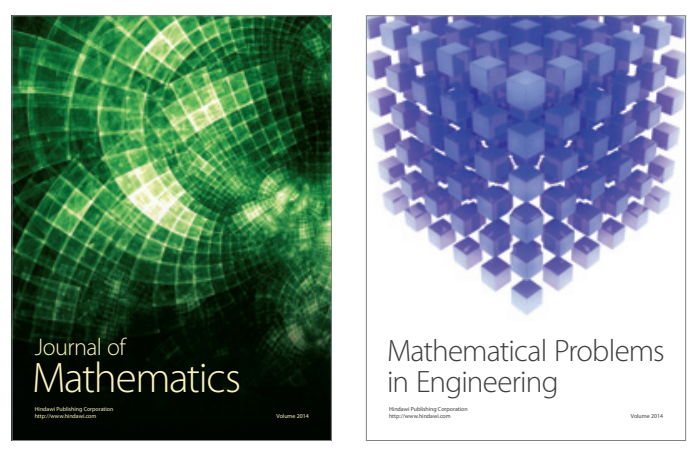

Mathematical Problems in Engineering
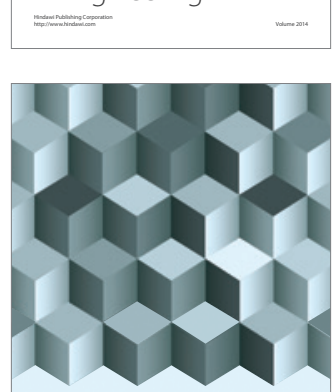

Journal of

Function Spaces
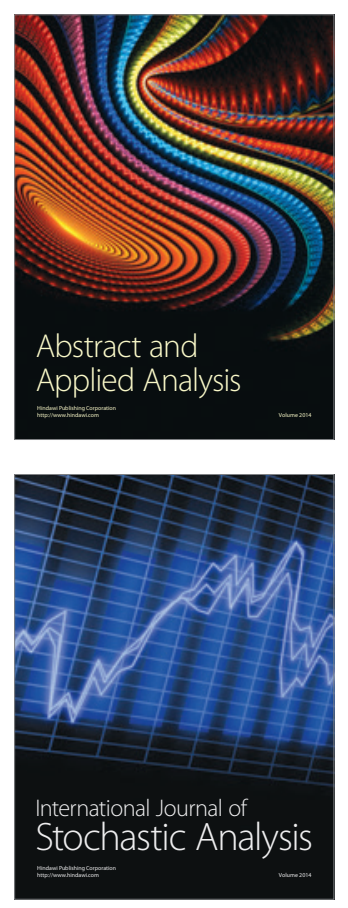

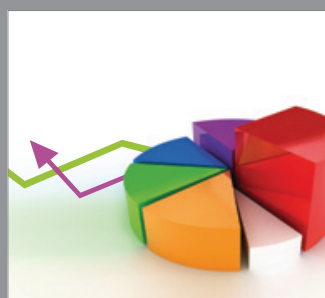

ournal of

Probability and Statistics

Promensencen
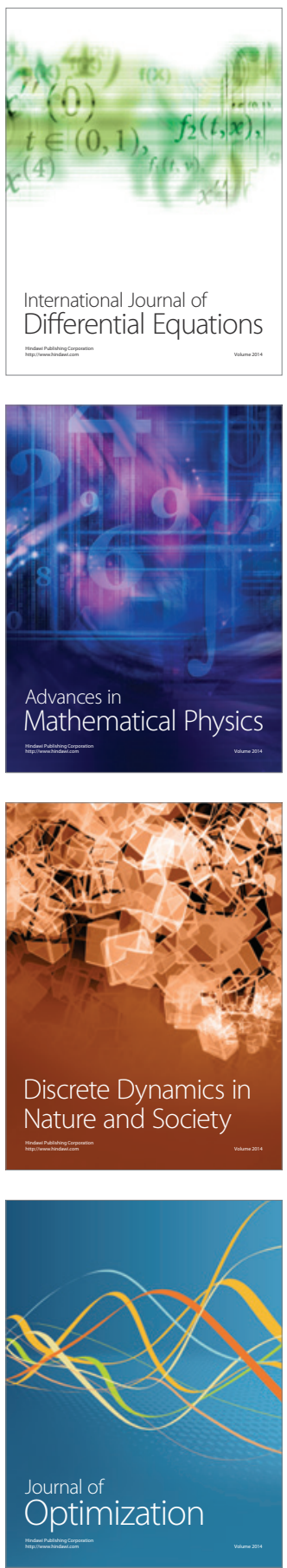